\title{
Utilização de processo Foto-Fenton no tratamento de águas contendo Benzeno-Tolueno-Xileno (BTX's)
}

\author{
F. L. NAVES ${ }^{1}$, M. B. PINTO ${ }^{1}$, L. M. de FARIA ${ }^{1}$, S. F. de AQUINO ${ }^{2}$, D. R. S. LIMA ${ }^{2}$ \\ ${ }^{1}$ Universidade Federal de São João Del Rei, Departamento de Engenharia Química e de Estatística \\ Email: fabianonavesengenheiro@gmail.com \\ ${ }^{2}$ Universidade Federal de Ouro Preto, Instituto de Ciências Exatas e Biológicas \\ Email: sergio@iceb.ufop.br
}

\begin{abstract}
RESUMO - Este trabalho tem por objetivo a avaliação da eficiência do processo fotoFenton no tratamento de uma solução sintética preparada a partir Benzeno, Tolueno e Xileno (BTX's) em água em concentrações $2 \mathrm{mM}$ respectivamente. Para tanto foi utilizado um reator tubular - Plug Flow Reactor (PFR) - de três módulos, sendo cada módulo concêntrico, contendo em seu interior uma lâmpada de UV de $15 \mathrm{~W}$ encamisada em tubo de vidro de borosilicado de uma polegada de diâmetro. Neste contexto foram avaliados a partir de uma matriz de Taguchi L9 os fatores, $\mathrm{pH}$, número de lâmpadas e concentração de $\mathrm{Fe}^{2+}$ e $\mathrm{H}_{2} \mathrm{O}_{2}$. A variável resposta do processo foi a carga orgânica detectada no equipamento medidor de carbono orgânico total (TOC) modelo TOC-L CSH. As amostras foram coletadas em intervalos de dez minutos durante uma batelada de uma hora. Após o carregamento do reator com a solução de BTX's uma amostra foi coletada a qual foi considerada como inicial, sem o efeito de qualquer tratamento posterior. A análise estatística foi realizada a partir do software Statistica ${ }^{\circledR}$. A maior redução da carga orgânica foi observada nas condições ótimas, sendo elas $\mathrm{pH} 3$, concentração de peroxido 1,5 $\mathrm{gL}^{-1}$, concentração de $\mathrm{Fe}^{2+} 0,1 \mathrm{gL}^{-1}$ e 3 lâmpadas operando. Porém vale ressaltar que a eficiência do processo utilizando duas ou três lâmpadas se mostrou muito próxima, efeito que pode ser aplicado na redução de custo de aplicação.
\end{abstract}

\section{INTRODUÇÃO}

A água ácida é uma das correntes mais preocupantes em uma refinaria, principalmente pela sua composição e seu caráter corrosivo. São consideradas como qualquer água de processo de uma refinaria que contenha sulfeto de hidrogênio $\left(\mathrm{H}_{2} \mathrm{~S}\right)$, como também amônia, fenóis, cianeto, óleo dissolvido, mercaptanas, hidrocarbonetos, sólidos suspensos, além de altos teores de benzeno, tolueno e xileno, remetendo assim à um teor elevado de carbono orgânico dissolvido (COD) e demanda química de oxigênio (DQO) (Gasparini, 2011).

O caráter tóxico do benzeno está relacionado diretamente com o seu potencial carcinogênico e mutagênico. Investigações ocupacionais em diferentes indústrias evidenciam que o benzeno desempenha um indubitável papel de risco aos humanos. A inalação de tolueno ou xilenos pode induzir distúrbios no modo de falar, na visão, audição, no controle dos músculos e outros, além de sugerirem a associação entre benzeno e xilenos e o surgimento de tumores cerebrais (Tiburtius; Peralta-Zamora, 2004).

Em função destes fatores a legislação tem se tornado cada vez mais restritiva. No Brasil, a portaria ${ }^{\circ} 1469 / 2000$ do Ministério da Saúde determina que os limites máximos permitidos para benzeno, tolueno e xilenos são de 5, 170 e $300 \mu \mathrm{g} / \mathrm{L}$, respectivamente, padrões este de potabilidade (Tiburtius; Peralta-Zamora, 2004). 


\subsection{Formas de Tratamento}

Dentre as várias formas de tratamento de resíduos líquidos, algumas podem ser omitidas devido a sua ineficiência a elevadas cargas recalcitrantes, como a presença de BTX's (Garg, Mishra; Chand, ). Portanto os processos oxidativos e os processos oxidativos avançados (POA's) tem se comportado como uma alternativa viável. Estes processos tem por base a produção de radicais hidroxilas através de um oxidante. Dentro da classe de POA's o processo foto- Fenton $\left(\mathrm{H}_{2} \mathrm{O}_{2} / \mathrm{Fe}^{2+} / \mathrm{UV}\right)$ tem sido um dos mais utilizados, devido sua faixa luminosa de trabalho compreendendo em parte da luz solar, diminuindo assim os custos de implementação do processo em scale - up (Gasparini, 2011).

O processo Fenton são reações compostas de peróxido, (geralmente peróxido de hidrogênio) com íons de ferro para a formação de espécies de oxigenadas ativas, com fim da oxidação de compostos orgânicos e inorgânicos, descoberto por H. J. H. Fenton em 1984 (H.J.H. Fenton, 1984). Atualmente tem se utilizado a reação de Fenton no tratamento de muitos compostos recalcitrantes, como um viés da solução de descontaminação de efluentes industriais que são impossibilitados de serem tratados em processos convencionais (Neyens, Baeyens, 2003). O tradicional mecanismo da reação de Fenton bem como as constantes de velocidade pode ser verificados nas equações de 1 a 9 (Sychev, Isak, 1995).

$$
\begin{gathered}
\mathrm{Fe}^{2+}+\mathrm{H}_{2} \mathrm{O}_{2} \rightarrow \mathrm{Fe}^{3+}+\mathrm{OH}^{-}+\mathrm{OH} \cdot \mathrm{K}_{1,1}=40-80 \mathrm{Lmol}^{-1} \mathrm{~S}^{-1}(1) \\
\mathrm{Fe}^{3+}+\mathrm{H}_{2} \mathrm{O}_{2} \rightarrow \mathrm{Fe}^{2+}+\mathrm{O}_{2} \mathrm{H} \cdot+\mathrm{H}^{+}, \mathrm{K}_{1,2}=9,1 \times 10^{-7} \mathrm{Lmol}^{-1} \mathrm{~S}^{-1}(2) \\
\mathrm{Fe}^{2+}+\mathrm{OH} \cdot \rightarrow \mathrm{Fe}^{3+}+\mathrm{OH}^{-}, \mathrm{K}_{1,3}=2,5-5 \mathrm{Lmol}^{-1} \mathrm{~s}^{-1}(3) \\
\mathrm{Fe}^{2+}+\mathrm{O}_{2} \mathrm{H} \cdot \rightarrow \mathrm{Fe}^{3+}+\mathrm{HO}_{2}^{-}, \mathrm{K}_{1,4}=0,72-1,5 \times 10^{-6} \mathrm{Lmol}^{-1} \mathrm{~s}^{-1}(4) \\
\mathrm{Fe}^{3+}+\mathrm{O}_{2} \mathrm{H} \cdot \rightarrow \mathrm{Fe}^{2+}+\mathrm{O}_{2}+\mathrm{H}^{+}, \mathrm{K}_{1,5}=0,33-2,1 \times 10^{-6} \mathrm{Lmol}^{-1} \mathrm{~S}^{-1}(5) \\
\mathrm{OH} \cdot+\mathrm{OH} \cdot \rightarrow \mathrm{H}_{2} \mathrm{O}_{2}, \mathrm{~K}_{1,6}=5-8 \times 10^{-9} \mathrm{Lmol}^{-1} \mathrm{~s}^{-1}(6) \\
\mathrm{OH}+\mathrm{H}_{2} \mathrm{O}_{2} \rightarrow \mathrm{O}_{2} \mathrm{H}+\mathrm{H}_{2} \mathrm{O}, \mathrm{K}_{1,7}=1,7-4,5 \times 10^{7} \mathrm{Lmol}^{-1} \mathrm{~S}^{-1}(7) \\
\mathrm{O}_{2} \mathrm{H} \cdot+\mathrm{O}_{2} \mathrm{H} \cdot \rightarrow \mathrm{H}_{2} \mathrm{O}_{2}+\mathrm{O}_{2}, \mathrm{~K}_{1,8}=0,8-2,2 \times 10^{6} \mathrm{Lmol}^{-1} \mathrm{~s}^{-1}(8) \\
\mathrm{O}_{2} \mathrm{H} \cdot+\mathrm{OH} \cdot \rightarrow \mathrm{H}_{2} \mathrm{O}+\mathrm{O}_{2}, \mathrm{~K}_{1,9}=1,4 \times 10^{10} \mathrm{Lmol}^{-1} \mathrm{~s}^{-1}(9)
\end{gathered}
$$

Na ausência ou presença de moléculas orgânicas, a reação de oxidação ocorre na decomposição do peroxido de hidrogênio na formação de oxigênio e água no processo. No entanto esta reação na degradação dos compostos orgânicos exige concentrações elevadas de peróxido de hidrogênio, aumentando assim consideravelmente o custo do tratamento (Xu; Wang, 2011). Vários trabalhos têm sido publicados na área de tratamento de efluentes líquidos utilizando como processo o foto Fenton. Composto estes na maior parte recalcitrante que não podem ser degradados via processos convencionais. Tratamento de compostos derivados de fenol (Akin Karci et al., 2014); Tratamento de compostos derivados de medicamentos que tem a capacidade de alterações hormonais aos seres humanos (Bizani; Veloutsou, 2014).

\section{Materiais e Métodos}

\subsection{Reagentes Químicos}

Os reagentes benzeno, tolueno e xileno foram adquiridos a partir do um padrão analítico da Sigma-Aldrich com $98 \%$ de pureza bem como as soluções foram preparados com água ultra 
pura Millipore. O peroxido de hidrogênio utilizado possui a concentração de $30 \% \mathrm{~m} / \mathrm{v}$ adquirido pela Vetec bem como o sulfato ferroso heptahidratado com pureza de 98,6\%.

\subsection{PROCEDIMENTO EXPERIMENTAL}

Os experimentos foram realizados em um reator Plug Flow Reactor (PFR) composto de três módulos, cada um com um volume útil de aproximadamente 3 litros. O reator é equipado com três lâmpadas de Ultravioleta com potência unitária de $15 \mathrm{~W}$. O interior de cada módulo do reator possui um tubo de uma polegada de borosilicato, o qual possui a finalidade de anular o contato do bulbo da luz UV com a solução de BTX's que circunda a parte útil do reator. A reação aconteceu na temperatura de $32 \pm 8$ graus Celsius controlada por um termômetro inserido no taque de reação. As amostras foram coletadas em intervalos de dez minutos e acondicionadas em sistema de refrigeração a $8 \pm 2$ graus Celsius, para evitar a propagação da reação com o restante de peróxido de hidrogênio presente. A variável resposta do processo de tratamento foi a degradação da carga orgânica medida a partir do carbono orgânico total que foi determinado pelo equipamento modelo TOC-L CSH da Shimatzu, a partir da diferença entre o carbono total e o carbono inorgânico. A avaliação da eficiência na degradação da matéria orgânica no processo foi feita a partir de uma matriz de Taguchi L9 composta de nove experimentos, conforme ilustra a Tabela 1.

Tabela 1- Condições Experimentais

\begin{tabular}{|c|c|c|c|}
\hline Fatores & Nível 1 & Nível 2 & Nível 3 \\
\hline $\mathrm{pH}$ & 2 & 3 & 5 \\
\hline $\mathrm{H} 2 \mathrm{O} 2\left(\mathrm{gL}^{-1}\right)$ & 0,2 & 0,5 & 1,5 \\
\hline $\mathrm{Fe}^{2+}\left(\mathrm{gL}^{-1}\right)$ & 0,2 & 0,1 & 0,3 \\
\hline $\mathrm{Luz}$ & 1 & 2 & 3 \\
\hline
\end{tabular}

\subsection{DESIGN EXPERIMENTAL}

Um design experimental robusto de Taguchi foi implementado para a averiguação dos parâmetros no foto-Fenton, na degradação dos compostos orgânicos presentes na solução. A escolha do método de Taguchi foi devido à avaliação robusta do processo, bem como a possibilidade de determinar o comportamento de cada parâmetro em função dos seus níveis averiguando assim a melhor condição experimental. A matriz de Taguchi esta apresentada na Tabela 2.

Tabela 2- Matriz de Taguchi L9

\begin{tabular}{|c|c|c|c|c|}
\hline Experimentos & $\mathrm{pH}$ & $\mathrm{H}_{2} \mathrm{O}_{2}\left(\mathrm{gL}^{-1}\right)$ & $\mathrm{Fe}^{2+}\left(\mathrm{gL}^{-1}\right)$ & Luz \\
\hline 1 & $2(1)$ & $0,2(1)$ & $0,2(1)$ & Uma lâmpada (1) \\
\hline 2 & $2(1)$ & $0,5(2)$ & $0,1(2)$ & Duas Lâmpadas (2) \\
\hline 3 & $2(1)$ & $1,5(3)$ & $0,3(3)$ & Três Lâmpadas (3) \\
\hline
\end{tabular}




\begin{tabular}{|c|c|c|c|c|}
\hline 4 & $3(2)$ & $0,2(1)$ & $0,1(2)$ & Três Lâmpadas (3) \\
\hline 5 & $3(2)$ & $0,5(2)$ & $0,3(3)$ & Uma lâmpada (1) \\
\hline 6 & $3(2)$ & $1,5(3)$ & $0,2(1)$ & Duas Lâmpadas (2) \\
\hline 7 & $5(3)$ & $0,2(1)$ & $0,3(3)$ & Duas Lâmpadas (2) \\
\hline 8 & $5(3)$ & $0,5(2)$ & $0,2(1)$ & Três Lâmpadas (3) \\
\hline 9 & $5(3)$ & $1,5(3)$ & $0,1(2)$ & Uma lâmpada (1) \\
\hline
\end{tabular}

O preenchimento da matriz de Taguchi acima foi feita numericamente pelos níveis descritos na Tabela 1, onde cada experimento executado consta de uma combinação de cada fator com seus respectivos níveis pré-estabelecidos.

\section{RESULTADOS E DISCUSSÃO}

A aplicação do processo foto Fenton no tratamento da solução de BTX's no reator PFR foi estudada a fim de determinar as melhores condições experimentais com intuito de um posterior scale up, principalmente na indústria petroquímica.

Todavia as análises estão envolvidas em determinar as melhores condições experimentais avaliadas segundo matriz de Taguchi L9, análise de Variâncias (ANOVA) bem como intepretação dos resíduos segundo a normalidade do processo em função dos valores preditos e observados de redução do carbono orgânico total. A Tabela 3 a seguir mostra os valores de degradação da solução de BTX's em função dos fatores e níveis estipulados.

Tabela 3- Matriz Taguchi com resultados alcançados

\begin{tabular}{|c|l|c|c|l|c|c|c|c|}
\hline Ensaio & Ph & $\mathbf{H}_{2} \mathbf{O}_{2}\left(\mathbf{g L}^{-\mathbf{1}}\right)$ & $\mathbf{F e}^{\mathbf{2 +}}\left(\mathbf{g L}^{-\mathbf{1}}\right)$ & $\mathbf{L u z}$ & $\begin{array}{c}\mathbf{\%} \\
\text { Redução } \\
\text { TOC }\end{array}$ & $\begin{array}{c}\text { Valor } \\
\text { Observado }\end{array}$ & $\begin{array}{c}\text { Valor } \\
\text { Predito }\end{array}$ & Resíduos \\
\hline 1 & $2(1)$ & $0,2(1)$ & $0,2(1)$ & Uma lâmpada (1) & 50,5 & 50,50 & 48,65889 & 1,8411 \\
\hline 2 & $2(1)$ & $0,5(2)$ & $0,1(2)$ & Duas Lâmpadas (2) & 61,8 & 61,80 & 61,60889 & 0,1911 \\
\hline 3 & $2(1)$ & $1,5(3)$ & $0,3(3)$ & Três Lâmpadas (3) & 76,4 & 76,40 & 74,55889 & 1,8411 \\
\hline 4 & $3(2)$ & $0,2(1)$ & $0,1(2)$ & Três Lâmpadas (3) & 48,4 & 48,40 & 61,56056 & $-13,3606$ \\
\hline 5 & $3(2)$ & $0,5(2)$ & $0,3(3)$ & Uma lâmpada (1) & 45,5 & 45,50 & 46,68056 & $-1,1806$ \\
\hline 6 & $3(2)$ & $1,5(3)$ & $0,2(1)$ & Duas Lâmpadas (2) & 63,35 & 63,35 & 56,55556 & 6,7944 \\
\hline 7 & $5(3)$ & $0,2(1)$ & $0,3(3)$ & Duas Lâmpadas (2) & 57,5 & 57,50 & 46,83222 & 10,6678 \\
\hline 8 & $5(3)$ & $0,5(2)$ & $0,2(1)$ & Três Lâmpadas (3) & 59,4 & 59,40 & 56,70722 & 2,6928 \\
\hline 9 & $5(3)$ & $1,5(3)$ & $0,1(2)$ & Uma lâmpada (1) & 32,14 & 32,14 & 41,62722 & $-9,4872$ \\
\hline
\end{tabular}

A melhor condição experimental foi velada pelo experimento 3 de acordo com os fatores: $\mathrm{pH}$, concentração de peróxido de hidrogênio, concentração de íons e número de lâmpadas utilizadas com seus respectivos níveis: $2,1,5 \mathrm{gL}^{-1}, 0,3 \mathrm{gL}^{-1}$ e 3 lâmpadas ligadas. A avaliação do pH no nível 3 está descrita na literatura, Monteagudo, J.M et al., (2010); Rodríguez-Gil et al., (2010); Trovó et al., (2013); Veloutsou, S Bizani, E Fytianos (2014). Para valores de pH elevados ou acimas de 3 , pode ocorrer a precipitação dos íons de ferro presentes em solução, diminuído assim o efeito da formação de radicais hidroxilas presentes e consequentemente diminuindo a eficiência na degradação dos compostos orgânicos presentes. $\mathrm{O}$ efeito do $\mathrm{pH}$ pode ser visto na Figura 1. 


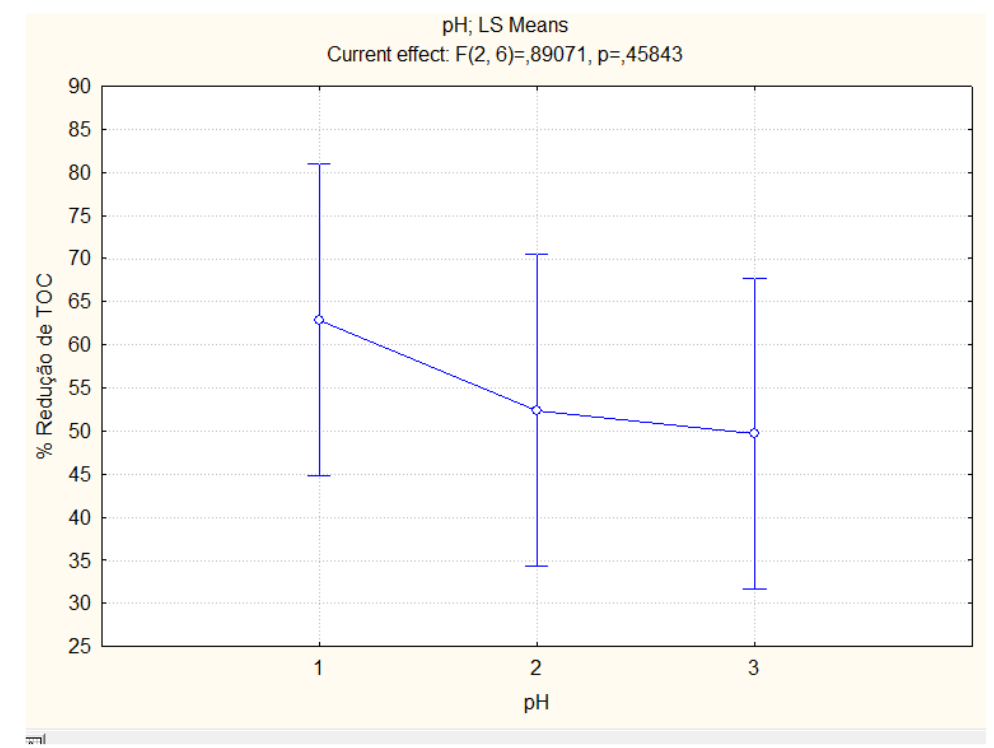

Figura 1- Efeito do pH na na redução do TOC

O efeito da concentração de peroxido de hidrogênio está diretamente relacionado com o percentual de radicais hidroxilas formado a partir deste oxidante. No entanto pelo mecanismo mostrado nas equações de 1 a 9, pode acontecer a recombinação dos pares de radicais hidroxilas para a formação de moléculas de peroxido de hidrogênio. Este fato diminui o rendimento reacional, mesmo o poder oxidante do peroxido de hidrogênio sendo 1,77 $\mathrm{V}$ o radical hidroxila supera o mesmo com um potencial de $2,81 \mathrm{~V}$. Portanto a melhor concentração de peroxido de hidrogênio no tratamento em questão neste trabalho, se encontra no nível mais elevado com valor de $1,5 \mathrm{gL}^{-1}$. Pode-se ainda verificar que do nível mais baixo (1) para o nível intermediário (2) houve uma ascendência acentuada na degradação vislumbrando que existe para a determinada concentração de BTX's há uma efetiva diferença de degradação entre os níveis em questionamento. A comparação entre a concentração deste oxidante entre os níveis (2) e (3) nos mostra que a inclinação diminui bem comparada anteriormente. Porém podemos denotar que a concentração de peróxido de hidrogênio no nível (3) está próxima da ideal como mostrado na Figura 2.

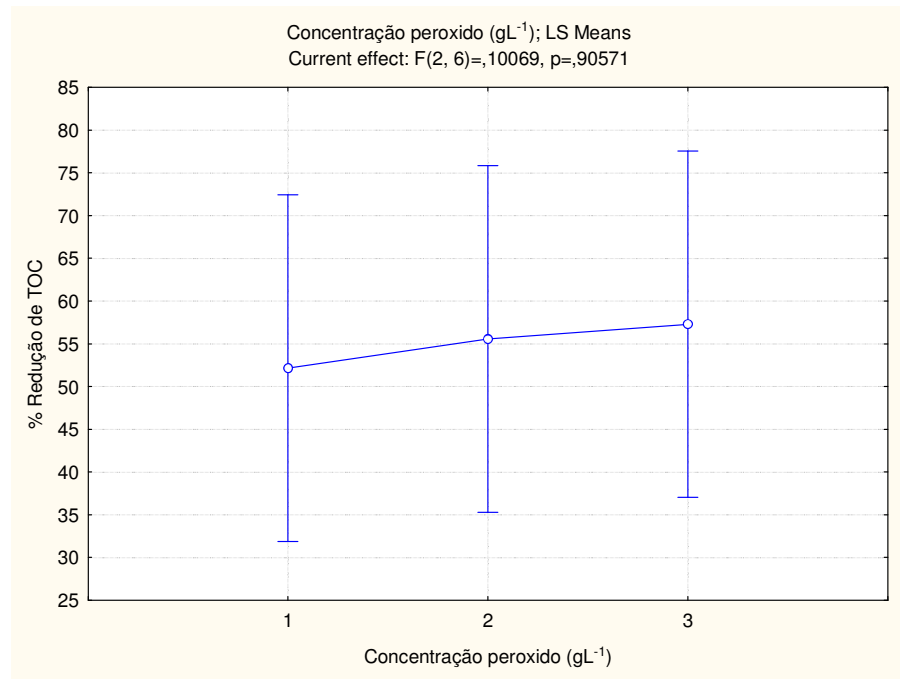

Figura 2- Efeito da concentração de peróxido na redução do TOC 
Os teores de íons de ferro presentes em solução estão relacionados com a relação entre a concentração de peroxido de hidrogênio discutida anteriormente. No entanto concentrações de íons de ferro não estequiométricas podem levar uma parte dos íons a precipitação pelo efeito do íon comum. Este efeito consequentemente diminuirá a eficiência do processo visto que os íons possuem a necessidade de estarem presentes em solução. A relação entre a concentração destes íons presentes em solução e a redução do TOC pode ser vista na Figura 4.

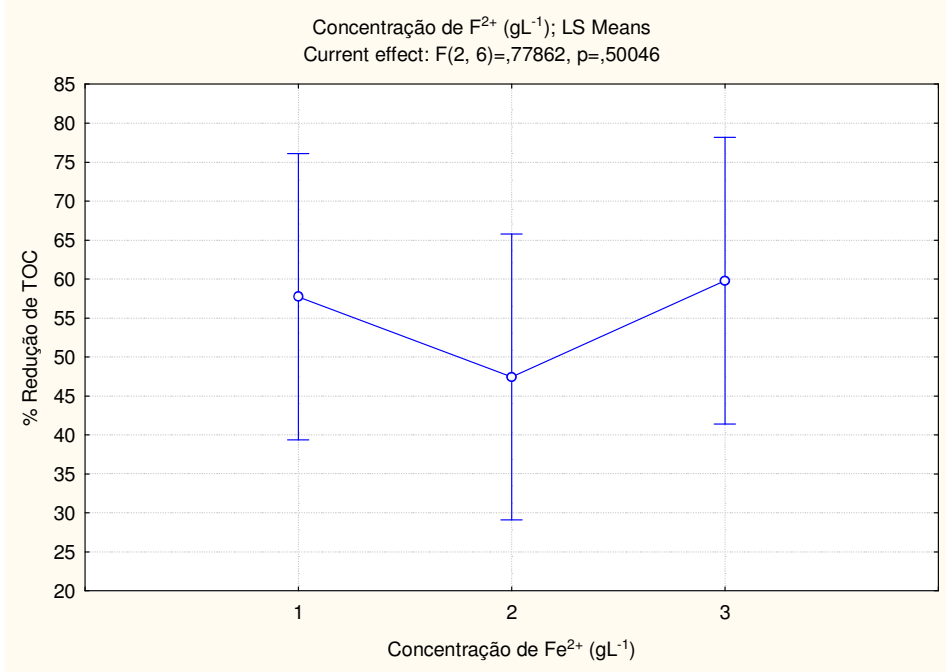

Figura 3- Efeito da concentração de íos Ferro na redução do TOC

visto que maior a taxa de luminosidade melhor o rendimento do processo de degradação. No entanto o objetivo principal da variação da intensidade luminosa no interior do reator está em determinar o nível máximo de luminosidade que corresponde a melhor eficiência e menor custo dispendido. Pode-se observar pela Figura 5 que a utilização de três lâmpadas apesar de maior intensidade luminosa, para o tempo espacial do reator utilizado não se mostrou mais eficiente que para a utilização de somente duas lâmpadas.

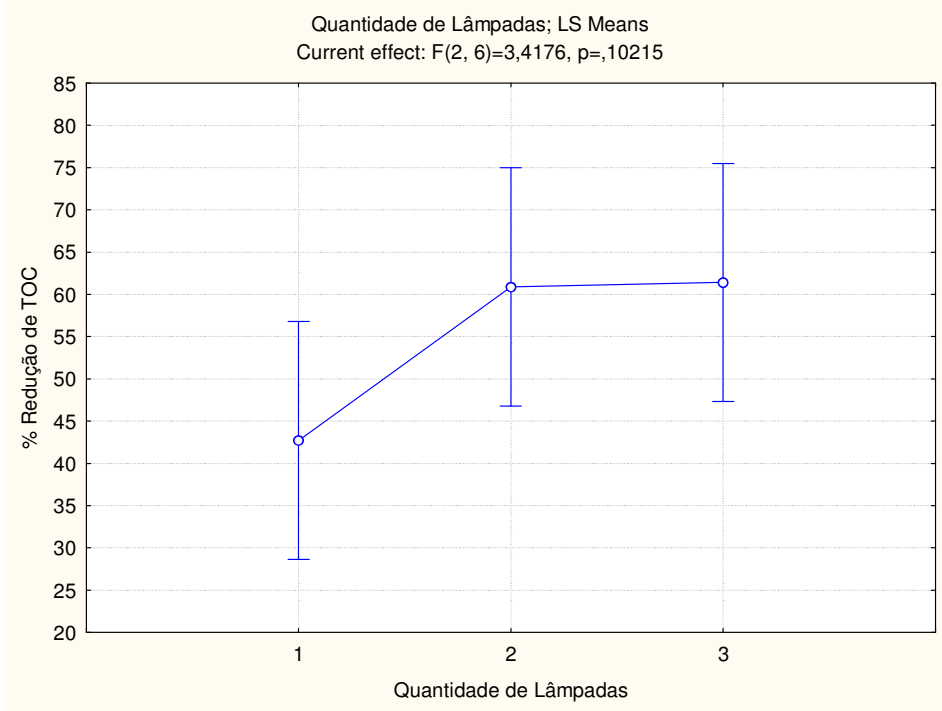

Figura 4- Efeito da intensidade Luminosa na redução do TOC 
De forma global podemos representar na Figura 6, todos os efeitos em relação a média bem como seus níveis avaliados na degradação direta do BTX's a partir da redução do TOC.

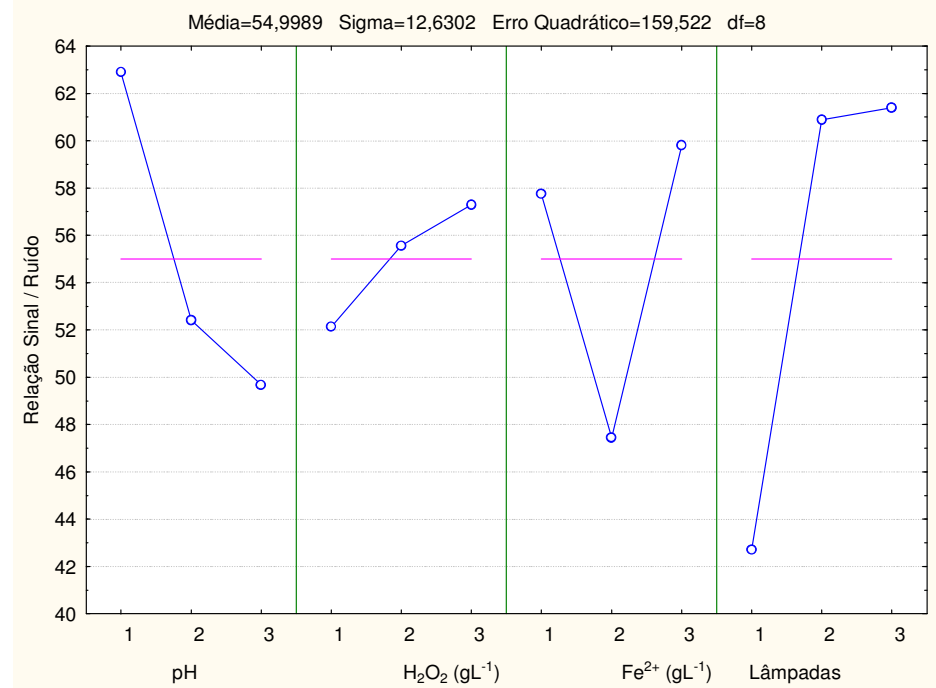

Figura 5- Relação sinal ruído dos fatores e seus respectivos níveis

O comportamento dos resíduos datados na Tabela 3 também é observado em uma distribuição normal, na Figura 7, fato esse que evidencia uma variância constante no decorrer do processo experimental.

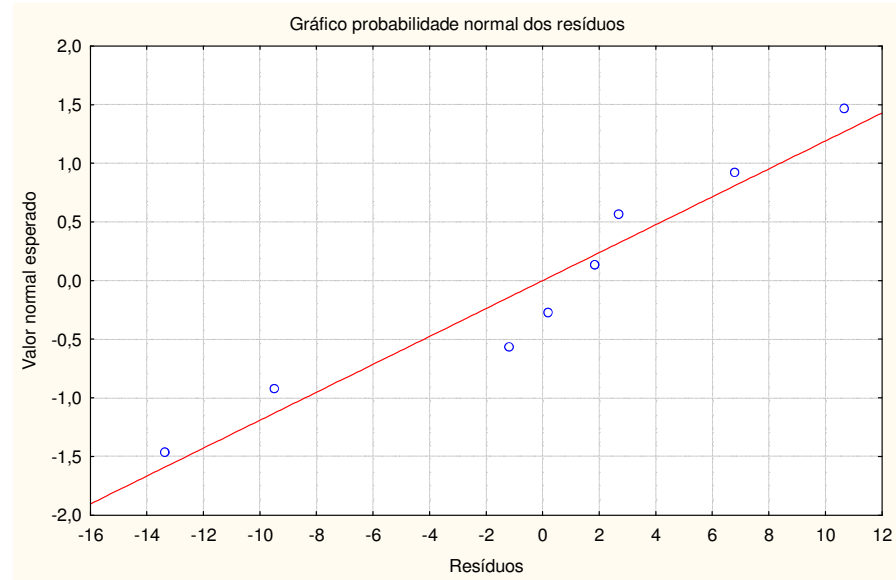

Figura 6- Relação dos resíduos na distribuição normal de probabilidade

\section{CONCLUSÃO}

Pode-se avaliar a partir dos dados obtidos experimentalmente, que o processo de degradação da solução de BTX's a partir do processo Foto-Fenton foi eficiente com remoção da carga orgânica em torno de $80 \%$ em tratamento com bateladas de 1 hora. Essa redução se enquadraria no padrão de descarte estabelecido pelo CONAMA 256. 


\section{REFERÊNCIAS BIBLIOGRÁFICAS}

BABUPONNUSAMI, A., \& MUTHUKUMAR, K. (2014). A review on Fenton and improvements to the Fenton process for wastewater treatment. Journal of Environmental Chemical Engineering, 2(1), 557-572. doi:10.1016/j.jece.2013.10.011

GASPARINI, M. C. Águas de Processo da REPLAN: Qualidade e Aplicação de Processos Oxidativos Avançados. 2011. Dissertação. (Mestrado em Engenharia Civil). Faculdade de Engenharia Civil, Arquitetura e Urbanismo, Universidade Estadual de Campinas, 2011.

KARCI, A., ARSLAN-ALATON, I., BEKBOLET, M., OZHAN, G., \& ALPERTUNGA, B. (2014). H2O2/UV-C and Photo-Fenton treatment of a nonylphenol polyethoxylate in synthetic freshwater: Follow-up of degradation products, acute toxicity and genotoxicity. Chemical Engineering Journal, 241, 43-51. doi:10.1016/j.cej.2013.12.022

MONTEAGUDO, J. M., DURÁN, a., MARTÍN, I. S., \& AGUIRRE, M. (2010). Effect of light source on the catalytic degradation of protocatechuic acid in a ferrioxalate-assisted photoFenton process. Applied Catalysis B: Environmental, 96(3-4), 486-495. doi:10.1016/j.apcatb.2010.03.009

RODRÍGUEZ-GIL, J. L., CATALÁ, M., ALONSO, S. G., MAROTO, R. R., VALCÁRCEL, Y., SEGURA, Y., ... MARTÍNEZ, F. (2010). Heterogeneous photo-Fenton treatment for the reduction of pharmaceutical contamination in Madrid rivers and ecotoxicological evaluation by a miniaturized fern spores bioassay. Chemosphere, 80(4), 381-8. doi:10.1016/j.chemosphere.2010.04.045

TIBURTIUS, E. R. L; PERALTA-ZAMORA, P. Degradação de BTX's via processos oxidativos avançados. 2004. Departamento de Química, Universidade Federal do Paraná.

TROVÓ, A. G., SILVA, T. F. S., GOMES, O., MACHADO, A. E. H., BORGES, W., MULLER, P. S., \& D, D. (2013). Chemosphere Degradation of caffeine by photo-Fenton process: Optimization of treatment conditions using experimental design, 90, 170-175. doi:10.1016/j.chemosphere.2012.06.022

VELOUTSOU, S., BIZANI, E., \& FYTIANOS, K. (2014). Photo-Fenton decomposition of $\beta$ blockers atenolol and metoprolol; study and optimization of system parameters and identification of intermediates. Chemosphere. doi:10.1016/j.chemosphere.2013.12.031 